\title{
Cirurgia Laparoscópica do Refluxo Gastroesofágico na Criança
}

\author{
Laparoscopic Surgery of Gastroesophageal Reflux in Children
}

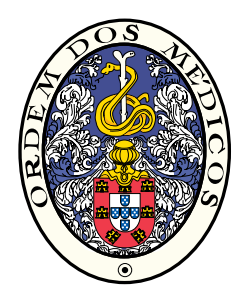

\author{
Ana DIAS ${ }^{1}$, Marina AMARAL ${ }^{2}$, Eunice TRINDADE ${ }^{3}$, Jorge Amil DIAS ${ }^{3}$, Miguel CAMPOS ${ }^{2}$, José ESTEVÃO-COSTA $^{2}$ \\ Acta Med Port 2013 Nov-Dec;26(6):705-710
}

\section{RESUMO}

Introdução: O refluxo gastroesofágico na criança é frequente e geralmente inócuo. Contudo, perante doença de refluxo gastroesofágico pode impor-se o tratamento cirúrgico, o qual pode ser realizado por via clássica ou laparoscópica. No presente estudo pretende-se avaliar a exequibilidade, eficácia e segurança da cirurgia antirrefluxo (fundoplicatura de Nissen) por via laparoscópica com ênfase no recém-nascido, lactente e primeira infância.

Material e Métodos: Estudaram-se as crianças com refluxo gastroesofágico propostas para cirurgia por via laparoscópica entre janeiro 2006 e dezembro 2012 num Serviço de Cirurgia Pediátrica de um Hospital universitário. Realizou-se uma análise descritiva e comparativa por faixa etária (grupo I: <1 ano, grupo II: 1-3 anos, grupo III: > 3 anos) e presença de comorbilidades. Foram avaliados os seguintes parâmetros: demografia, sintomatologia, comorbilidades, indicações operatórias, perioperatório e evolução pós-operatória. Foi assumido um nível de significância de $5 \%$.

Resultados: Foram propostas 55 crianças, das quais 12 do grupo I, 15 do grupo II e 28 do grupo III. 50,9\% apresentavam comorbilidades (21 com doença do sistema nervoso central e sete com atresia esofágica). Em 52 (94,5\%) casos, a cirurgia foi exequível exclusivamente por via laparoscópica. A duração do procedimento foi de $167 \pm 42$ minutos, sem diferenças significativas entre grupos etários, mas superior na doença do sistema nervoso central. Com um seguimento de 35,5 $\pm 23,9$ meses, ocorreram as seguintes complicações: síndrome de gas-bloat, dumping e disfagia em oito casos $(15,7 \%)$ e hérniaparaesofágica em dois casos $(3,9 \%)$, sem diferenças significativas entre os grupos; verificou-se recidiva clínica com reoperação em dois casos.

Conclusão: A fundoplicatura de Nissen laparoscópica é um procedimento exequível, eficaz e com baixa morbilidade, inclusive em recém-nascidos, lactentes e na primeira infância.

Palavras-chave: Criança; Fundoplicatura; Laparoscopia; Procedimentos Cirúrgicos Minimamente Invasivos; Portugal; Refluxo Gastroesofágico.

\section{ABSTRACT}

Introduction: Gastroeshophageal reflux is a common but usually innocuous condition in children. However, in the presence of Gastroeshophageal reflux disease, the surgical treatment (open or laparoscopic) may be indicated. This study aimed to evaluate the feasibility, effectiveness and safety of laparoscopic antireflux surgery (Nissen fundoplication) with emphasis in infants.

Material and Methods: Children with gastroeshophageal reflux proposed for laparoscopic antireflux surgery between January 2006 and December 2012 in a pediatric surgery department of a university hospital were studied. A descriptive and comparative analysis was conducted with regard to age (group I: <1 year, group II: 1-3 years, group III: > 3 years) and presence of comorbidities. Demographics, symptoms, comorbidities, operative indications, perioperative and postoperative outcome were assessed. The statistical significance level was set at $5 \%$.

Results: 55 children were proposed for surgery: 12 of group I, 15 group of II and 28 of group III. $50.9 \%$ had comorbidities (21 central nervous system disease and 7 esophageal atresia). In 52 (94.5\%) cases, antireflux surgery was performed by laparoscopy. The procedure lasted $167 \pm 42$ minutes, with no significant differences according to age groups, although higher in those with central nervous system disease. With a follow-up of $35.5 \pm 23.9$ months, the following complications occurred: gas-bloat syndrome, dumping or dysphagia in 8 cases $(15.7 \%)$ and paraesophageal hernia in 2 cases $(3.9 \%)$, with no significant differences between groups; there was clinical recurrence with reoperation in 2 cases.

Conclusion: Laparoscopic Nissen fundoplication is a feasible and effective procedure, with minimal morbidity, including in infancy.

Keywords: Child; Gastroesophageal Reflux; Laparoscopy; Fundoplication; Portugal; Surgical Procedures, Minimally Invasive.

\section{INTRODUÇÃO}

A doença por refluxo gastroesofágico (DRGE) é um dos motivos mais comuns de consulta em centros pediátricos de referência. ${ }^{1}$ Apesar de o refluxo gastroesofágico (RGE) ser geralmente inócuo, pode resultar numa evolução grave, nomeadamente para esofagite, o que realça a importância do diagnóstico e tratamento precoces..$^{2-6}$

A abordagem da DRGE deve ser estratificada, o que poderá incluir alterações no estilo de vida, terapêuticas far- macológicas e cirurgia antirefluxo. ${ }^{7,8} \mathrm{Em}$ teoria, o tratamento cirúrgico da DRGE deverá ser considerado após falência do tratamento médico. ${ }^{6,9}$ No entanto, para alguns autores, a presença de complicações tais como estenose péptica, esófago de Barrett e eventos aparentemente ameaçadores de vida (ALTE) podem constituir indicações operatórias $a b$ initio. $^{6,9-13}$

A cirurgia antirrefluxo é um procedimento frequente-

\footnotetext{
1. Faculdade de Medicina. Universidade do Porto. Porto. Portugal.

2. Serviço de Cirurgia Pediátrica. Hospital S. João e Faculdade de Medicina. Universidade do Porto. Porto. Portugal.

3. Unidade de Gastrenterologia Pediátrica. Hospital S. João. Porto. Portugal.

Recebido: 20 de Março de 2013 - Aceite: 23 de Setembro de 2013 | Copyright @ Ordem dos Médicos 2013
} 
mente realizado em centros de cirurgia pediátrica terciários. ${ }^{6,8,12-18} \mathrm{~A}$ abordagem operatória pode ser realizada por via clássica (laparotomia) ou por via laparoscópica, embora a embora a última seja assumida pela grande maioria dos autores como gold standard. $., 9,10,16,17,19$

Vários estudos têm demonstrado a eficácia e segurança da fundoplicatura por via laparoscópica realizada em crianças, com as vantagens inerentes a este tipo de abordagem. ${ }^{9,12,14,16}$ No entanto, a sua exequibilidade, eficácia e segurança continuam a ser debatidas, sobretudo na primeira infância, no lactente e no recém-nascido. ${ }^{6,13,14}$

No presente estudo pretende-se caracterizar uma população pediátrica com RGE submetido a tratamento cirúrgico, com o objetivo principal de avaliar a exequibilidade, eficácia e morbilidade da cirurgia laparoscópica em recém-nascidos, lactentes e na primeira infância.

\section{MATERIAL E MÉTODOS}

Analisaram-se os casos de DRGE propostos para cirurgia por via laparoscópica num serviço de Cirurgia Pediátrica de um hospital universitário, entre janeiro de 2006 e dezembro de 2012. A técnica operatória consistia de uma fundoplicatura total (floppy Nissen), associada à colocação de botão de gastrostomia nos casos com disfunção da deglutição. As indicações operatórias foram: falência do tratamento médico ou presença de estenose péptica, esófago de Barret ou episódios de ALTE.

Após aprovação pela Comissão de Ética da instituição hospitalar foram recolhidos dados relativos a: demografia (idade, sexo, peso), tipo de sintomatologia, comorbilidades (doença do sistema nervoso central (SNC), atresia esofágica $(\mathrm{AE})$ ), indicação operatória, perioperatório (tipo de intervenção cirúrgica, duração do procedimento operatório, procedimentos associados, complicações intraoperatórias e duração de internamento) e evolução pós-operatória (eficácia e complicações).

Para além da análise descritiva, efetuou-se uma análise comparativa entre três grupos de acordo com a faixa etária, nomeadamente: Grupo I (recém-nascidos e lactentes, 0-12 meses), Grupo II (primeira Infância, 12-36 meses) e Grupo III (3-18 anos).

Efetuou-se ainda uma análise comparativa para avaliar a influência de fatores como a presença de doença do SNC, colocação de botão de gastrostomia e antecedentes de $A E$.

Os valores apresentados correspondem às médias \pm desvio padrão para as variáveis contínuas e às frequências para as variáveis categóricas. $\mathrm{Na}$ análise estatística de variáveis contínuas foram utilizados os testes $t$-student (independente), teste ANOVA e teste de Kruskal-Wallis conforme adequado, sendo a normalidade das variáveis analisada usando o teste de Kolmogorov-Smirnov. As variáveis categóricas foram analisadas com o teste Qui-quadrado ou teste exato de Fisher. O nível de significância $(p)$ considerado foi de $5 \%$. A análise estatística foi realizada utilizando o software IBM SPSS Statistics $20^{\circledR}$.

\section{RESULTADOS}

Cinquenta e cinco crianças foram propostas para cirurgia laparoscópica antirrefluxo: $12(21,8 \%)$ pertenciam ao grupo I (recém-nascidos e lactentes), 15 (27,3\%) ao grupo II (primeira infância) e 28 (50,9\%) ao grupo III (> três anos), cuja caracterização demográfica e clínica está descrita na Tabela 1.

Nesta série verificou-se um predomínio do sexo masculino em todos os grupos. O peso corporal variou entre $2,4 \mathrm{e}$ $60 \mathrm{~kg}$. A doença do SNC e os antecedentes de AE foram as comorbilidades mais frequentes, particularmente no grupo II e III. As crianças da primeira infância tinham a maior prevalência de comorbilidades (73,3\%).

No que respeita à forma de apresentação clínica, predominaram os sintomas digestivos. Nos lactentes, uma proporção substancial (50\%) apresentava sintomas respiratórios.

A indicação operatória mais frequente foi a DRGE refratária ao tratamento médico $(81,8 \%)$, com diferenças substanciais entre os grupos etários (Tabela 2). A presença de ALTE apenas ocorreu no grupo I e foi quantitativamente idêntica à DRGE refratária ao tratamento médico. Todas as crianças do grupo II foram propostas por falência do tratamento médico. A presença de estenose péptica e esófago de Barrett constituíram indicações ab initio apenas no grupo III.

O procedimento cirúrgico foi realizado exclusivamente por via laparoscópica em 52 (94,5\%) crianças (Fig. 1), sem diferenças estatisticamente significativas da frequência de conversão para laparotomia entre os vários grupos. As conversões deveram-se a avaria de material $(n=1)$ e impossibilidade técnica por bridas e/ou gastrostomia prévia $(n=2)$. A taxa de conversão para laparotomia também não foi significativamente afetada pela presença de doença do SNC [2/21 (9,5\%) vs $1 / 34(2,9 \%), p=0,323]$ ou pela presença de antecedentes de $\mathrm{AE}[0 / 7(0 \%)$ vs $3 / 48(6,2 \%), p=0,659]$.

Nos casos submetidos a cirurgia exclusivamente por via laparoscópica foi colocado botão de gastrostomia em 14 doentes $(26,9 \%)$, com uma proporção maior no grupo II e III (Tabela 3). A colocação de botão de gastrostomia foi significativamente mais alta nas crianças com doença do SNC [13/19 (68,4\%) vs 1/33 (3\%), $p<0,001]$.

A duração do procedimento laparoscópico foi de $167 \pm$ 42 minutos, sem diferenças significativas entre os grupos, com um mínimo de 95 minutos e um máximo de 315 minutos. A duração do procedimento foi maior nas crianças em que foi colocado botão de gastrostomia (186 \pm 49 vs 160 \pm 39 minutos, $p=0,056)$. A presença de doença do SNC aumentou significativamente a duração do procedimento operatório (190 \pm 482 vs $154 \pm 34$ minutos, $p=0$,002). Nas crianças com antecedentes de AE, a duração da cirurgia foi significativamente menor $(138 \pm 24$ vs $172 \pm 43$ minutos, $p=0,049$ ).

Ocorreram duas complicações intraoperatórias: uma laceração do cárdia no grupo I e uma laceração superficial do baço no grupo III, ambas corrigidas sem necessidade de conversão. 
Tabela 1 - Demografia, comorbilidades e sintomatologia

\begin{tabular}{|c|c|c|c|c|c|c|c|c|c|}
\hline & \multirow[b]{2}{*}{$N$} & \multirow[b]{2}{*}{$\begin{array}{c}\text { Idade } \\
\text { (meses) }\end{array}$} & \multirow[b]{2}{*}{$\begin{array}{c}\text { Sexo } \\
(M / F)\end{array}$} & \multirow[b]{2}{*}{$\begin{array}{l}\text { Peso } \\
(\mathrm{Kg})\end{array}$} & \multicolumn{2}{|c|}{ Comorbilidades } & \multicolumn{3}{|c|}{ Sintomas } \\
\hline & & & & & $\begin{array}{c}\text { dçSNC } \\
n(\%)\end{array}$ & $\begin{array}{c}\mathrm{AE} \\
n(\%)\end{array}$ & $\begin{array}{l}\text { Digestivos } \\
n(\%)\end{array}$ & $\begin{array}{c}\text { Respiratórios } \\
n(\%)\end{array}$ & $\begin{array}{c}\text { Ambos } \\
n(\%)\end{array}$ \\
\hline Grupo I & 12 & $5,3 \pm 4,2$ & $9(75 \%) / 3$ & $\begin{array}{c}5,7 \pm 2,3 \\
{[2,7-9,6]}\end{array}$ & $2(16,7 \%)$ & $0(0 \%)$ & $6(50 \%)$ & $5(41,7 \%)$ & $1(8,3 \%)$ \\
\hline Grupo II & 15 & $23,9 \pm 7,7$ & $9(60 \%) / 6$ & $\begin{array}{c}8,5 \pm 2,7 \\
{[2,4-12,5]}\end{array}$ & $6(40 \%)$ & $5(33,3 \%)$ & $9(60 \%)$ & $2(13,3 \%)$ & $4(26,7 \%)$ \\
\hline Grupo III & 28 & $98,6 \pm 53,9$ & $17(60,7 \%) / 11$ & $\begin{array}{c}25,4 \pm 16,2 \\
{[8-60]}\end{array}$ & $13(46,4 \%)$ & $2(7,1 \%)$ & $18(64,3 \%)$ & $2(7,1 \%)$ & $8(28,6 \%)$ \\
\hline Global & 55 & $\begin{array}{c}57,8 \pm 57,1 \\
{[0-205]}\end{array}$ & $35(63,6 \%) / 20$ & $\begin{array}{c}16,1 \pm 14,6 \\
{[2,4-60]}\end{array}$ & $21(38,2 \%)$ & $7(12,7 \%)$ & $33(60 \%)$ & $9(16,4 \%)$ & $13(23,6 \%)$ \\
\hline \multicolumn{10}{|c|}{$\begin{array}{l}\text { Legenda: Grupo I - lactentes; Grupo II - primeira infância; Grupo III - > } 3 \text { anos; M- masculino; F- feminino; ḑ̧SNC - doença do Sistema Nervoso Central; AE - atresia esofágica } \\
\text { Entre parêntesis reto- valores limite da amostra }\end{array}$} \\
\hline \multicolumn{10}{|c|}{ Tabela 2 - Indicação operatória } \\
\hline & & \multicolumn{3}{|c|}{$\begin{array}{l}\text { Ineficácia tratamento médico } \\
\qquad n(\%)\end{array}$} & \multicolumn{4}{|c|}{$\begin{array}{l}\text { Estenose péptica / esófago de Barrett } \\
\qquad n(\%)\end{array}$} & $\begin{array}{l}\text { ALTE } \\
n(\%)\end{array}$ \\
\hline \multicolumn{2}{|c|}{ Grupo I } & \multicolumn{3}{|c|}{$6(50 \%)$} & \multicolumn{3}{|c|}{$0(0 \%)$} & \multicolumn{2}{|r|}{$6(50 \%)$} \\
\hline \multicolumn{2}{|c|}{ Grupo II } & \multicolumn{3}{|c|}{$15(100 \%)$} & \multicolumn{3}{|c|}{$0(0 \%)$} & \multicolumn{2}{|r|}{$0(0 \%)$} \\
\hline \multicolumn{2}{|c|}{ Grupo III } & \multicolumn{3}{|c|}{$24(85,7 \%)$} & \multicolumn{3}{|c|}{$4(14,3 \%)$} & \multicolumn{2}{|r|}{$0(0 \%)$} \\
\hline \multicolumn{2}{|c|}{ Global } & \multicolumn{3}{|c|}{$45(81,8 \%)$} & \multicolumn{3}{|c|}{$4(7,3 \%)$} & \multicolumn{2}{|c|}{$6(10,9 \%)$} \\
\hline
\end{tabular}

Legenda: Grupo I - lactentes; Grupo II - primeira infância; Grupo III - > 3 anos; DRGE - Doença de refluxo gastroesofágico; ALTE- apparent life-threatening event

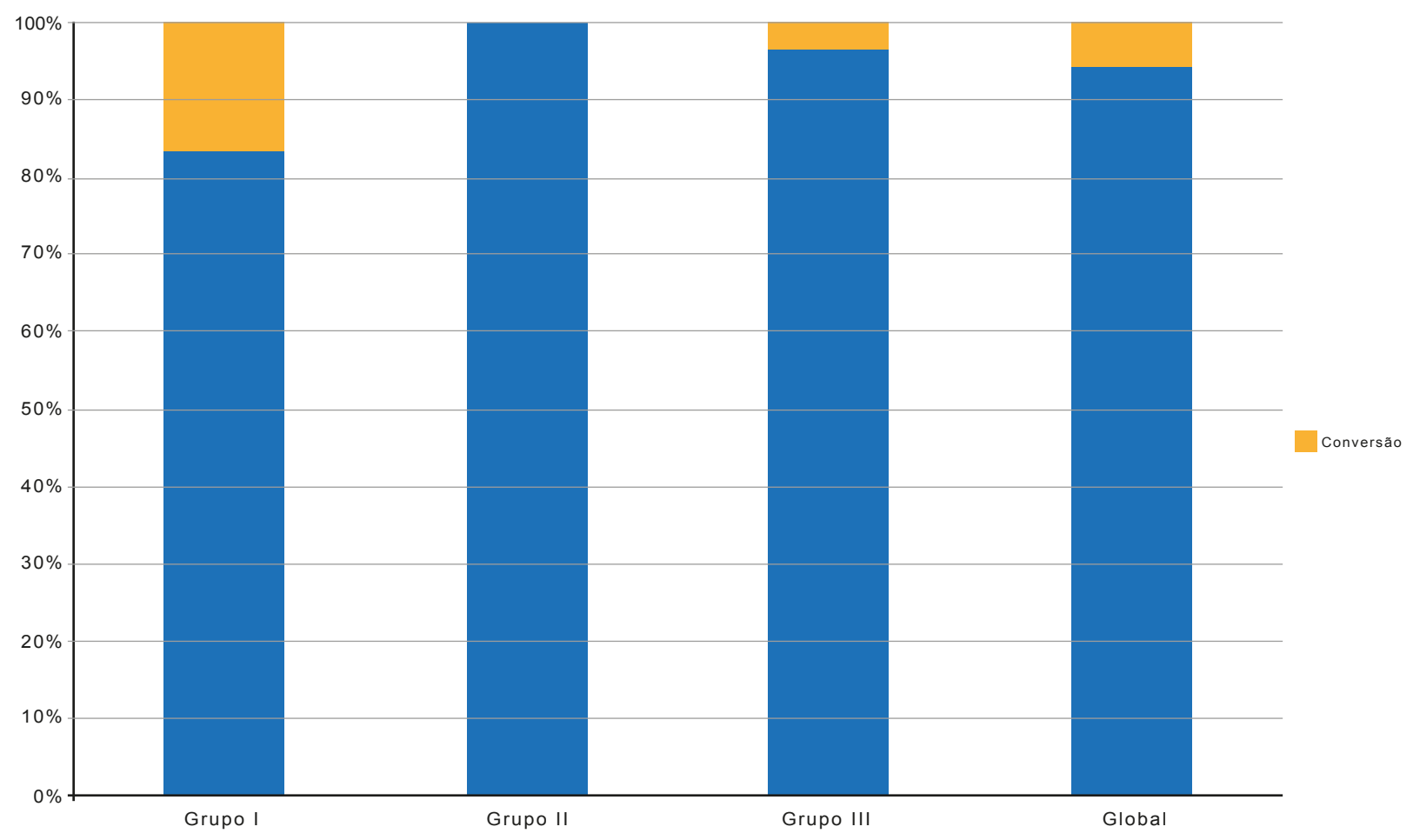

Figura 1 - Cirurgia laparoscópica antirrefluxo 
A duração do internamento foi de $3,9 \pm 2,6$ dias (variando entre 1 e 17 dias), sem diferenças estatisticamente significativas entre os três grupos ou entre crianças com e sem antecedentes de AE. A duração do internamento foi significativamente maior nas crianças com doença do SNC $(5,1 \pm 3,7$ vs $3,3 \pm 1,4$ dias, $p=0,024)$.

Uma criança do grupo III não foi seguida neste hospital, razão pela qual não foi considerada nos resultados descritos $(n=51)$. O tempo de seguimento dos doentes foi de $35,5 \pm 23,9$ meses (Tabela 4).

No grupo I ocorreram complicações em duas crianças: um dumping pós-operatório e uma síndrome gas-bloat. Todos os lactentes apresentavam bom estado geral, sem recidiva clínica, embora o tempo de seguimento fosse mais curto. No grupo II (primeira infância) ocorreu gas-bloat em uma criança e em duas foi detetada herniação paraesofágica. Esta última complicação foi responsável pela recidiva clínica da DRGE que motivou reoperação. No grupo III ocorreram três casos de síndrome de gas-bloat e dois de disfagia. Nestas crianças não se registaram recidivas clínicas.

Não se verificaram diferenças estatisticamente significativas na ocorrência de complicações, recidiva dos sintomas ou necessidade de reoperação entre os três grupos etários, assim como entre crianças com e sem doença do SNC ou entre crianças com e sem antecedentes de AE.

\section{DISCUSSÃO}

O RGE é geralmente inofensivo no lactente e nas crianças na primeira infância já que existem mecanismos fisiológicos que tornam a sua ocorrência ocasional inócua. ${ }^{20}$ No entanto, na presença de DRGE impõe-se uma atitude ativa dadas as possíveis complicações. ${ }^{4,15,20}$

A abordagem da DRGE pode implicar a realização de cirurgia antirrefluxo. As indicações operatórias não são unânimes. ${ }^{8,11,12,20} \mathrm{Na}$ presente série assumiram-se como indicações operatórias, para além da falência do tratamento médico, a presença $a b$ initio de estenose péptica, esófago de Barrett e episódios de ALTE. Embora seja defensável que a maturação da barreira antirrefluxo possa prevenir a ocorrência de episódios de ALTE, a esofagite, apesar de incomum nas crianças com menor idade, pode evoluir rapidamente para estenose e esófago de Barrett. 5,6,8-11,20 De realçar que as perspetivas do tratamento conservador/médico são mais sombrias em crianças com comorbilidades, nomeadamente doença do SNC e AE, nas quais o RGE tem uma prevalência $(65-70 \%$ e $30-80 \%$, respetivamente) e gravidade acrescidas. ${ }^{2,3,8,13,16,20,21}$

No que respeita à $\mathrm{AE}$, praticamente todos os componentes envolvidos na formação da barreira anti-refluxo podem estar comprometidos. O encurtamento do segmento esofágico abdominal, com diminuição do ângulo de His, relacionados com o repuxamento do segmento distal aquando do restabelecimento cirúrgico do trânsito esofágico, e as alterações da motilidade esofágica, com componente

Tabela 3 - Cirurgia laparoscópica

\begin{tabular}{|c|c|c|c|c|c|c|}
\hline & & Colocação de botão & Duração o & $\begin{array}{l}\text { cedimento } \\
\text { s) }\end{array}$ & Complicações & Duração \\
\hline & & $n(\%)$ & $\begin{array}{c}\text { Sem } \\
\text { gastrostomia }\end{array}$ & $\begin{array}{c}\text { Com } \\
\text { gastrostomia }\end{array}$ & $n(\%)$ & (dias) \\
\hline Grupo I & 10 & $1(10,0 \%)$ & $\begin{array}{c}145 \pm 29 \\
{[115-200]}\end{array}$ & 150 & $1(10 \%)$ & $3,3 \pm 1,5[2-7]$ \\
\hline Grupo II & 15 & $4(26,7 \%)$ & $\begin{array}{c}169 \pm 42 \\
{[95-265]}\end{array}$ & $\begin{array}{c}190 \pm 35 \\
{[162-240]}\end{array}$ & $0(0 \%)$ & $3,4 \pm 1,1[1-6]$ \\
\hline Grupo III & 27 & $9(33,3 \%)$ & $\begin{array}{c}163 \pm 40 \\
{[95-250]}\end{array}$ & $\begin{array}{c}188 \pm 57 \\
{[125-315]}\end{array}$ & $1(3,7 \%)$ & $4,52 \pm 3,4[2-17]$ \\
\hline Global & 52 & $14(26,9 \%)$ & $\begin{array}{c}160 \pm 39 \\
{[95-265]}\end{array}$ & $\begin{array}{c}186 \pm 49 \\
{[125-315]}\end{array}$ & $2(3,8 \%)$ & $3,96 \pm 2,61[1-17]$ \\
\hline
\end{tabular}

Legenda: Grupo I - lactentes; Grupo II - primeira infância; Grupo III - > 3 anos. Entre parêntesis reto- valores limite da amostra

Tabela 4 - Seguimento pós-operatório

\begin{tabular}{|c|c|c|c|c|c|c|}
\hline & \multirow{2}{*}{$N$} & \multirow{2}{*}{$\begin{array}{l}\text { Duração } \\
\text { (meses) }\end{array}$} & \multirow{2}{*}{$\begin{array}{c}\text { Recidiva clínica } \\
n(\%)\end{array}$} & \multicolumn{2}{|c|}{$\begin{array}{c}\text { Complicações } \\
n(\%)\end{array}$} & \multirow{2}{*}{$\begin{array}{c}\text { Reoperações } \\
n(\%)\end{array}$} \\
\hline & & & & Funcionais* & Hérnia paraesofágica & \\
\hline Grupo I & 10 & $10,8 \pm 5,9$ & $0(0 \%)$ & $2(20 \%)$ & $0(0 \%)$ & $0(0 \%)$ \\
\hline Grupo II & 15 & $46,2 \pm 19,8$ & $2(13 \%)$ & $1(6,7 \%)$ & $2(13,3 \%)$ & $2(13,3 \%)$ \\
\hline Grupo III & 26 & $38,9 \pm 24,4$ & $0(0 \%)$ & $5(19,2 \%)$ & $0(0 \%)$ & $0(0 \%)$ \\
\hline Global & 51 & $35,5 \pm 23,9$ & $2(3,9 \%)$ & $8(15,7 \%)$ & $2(3,9 \%)$ & $2(3,9 \%)$ \\
\hline
\end{tabular}

Legenda: Grupo I - lactentes; Grupo II - primeira infância; Grupo III - > 3 anos; *síndrome de gas-bloat, dumping e disfagia 
congénito e adquirido por lesão cirúrgica, são fatores que favorecem o desenvolvimento de RGE e das suas complicações. $6,8,13,22-24$

As crianças com doença do SNC têm também uma prevalência aumentada de DRGE, atribuindo-se uma origem multifatorial, nomeadamente disfunção do SNC, incoordenação laringofaríngea, dismotilidade esofágica, aumento da pressão intra-abdominal por espasticidade, atraso no esvaziamento gástrico, aumento da pressão intragástrica e escoliose, entre outros. ${ }^{8,13,25-27}$

Quando existe indicação para tratamento cirúrgico do RGE, e apesar de ainda não existir um gold standard, a opção pela cirurgia minimamente invasiva é crescente dadas as vantagens inerentes a este tipo de abordagem, tais como menor tempo de internamento, início mais precoce da alimentação oral e menor necessidade de analgesia pós-operatória, para uma eficácia aparentemente semeIhante à da cirurgia por via clássica. ${ }^{12,16,17}$

Uma área em discussão é a exequibilidade, segurança e eficácia da cirurgia minimamente invasiva no recém-nascido e lactente, podendo questionar-se o papel da cirurgia antirrefluxo laparoscópica. ${ }^{14,20}$

A ausência de diferenças significativas no que respeita à conversão para laparotomia, com uma alta taxa de procedimentos exclusivamente por via laparoscópica (94,5\%), na duração do procedimento e na frequência de complicações intraoperatórias entre os vários grupos etários analisados apoia a exequibilidade desta técnica nas crianças de menor idade. No que respeita à segurança, a taxa de complicações foram baixas (sem diferenças entre os grupos etários) e a duração do internamento equivalente nas diferentes faixas etárias, o que reforça o papel da cirurgia laparoscópica.

A eficácia no controlo dos sintomas foi de 96,1\%. Apesar das complicações (geralmente minor e transitórias), é importante ter em consideração que a cirurgia antirrefluxo controla os sintomas de uma maneira eficaz, com melhoria da qualidade de vida dos doentes e dos seus cuidadores. $6,16,18,20$

Independentemente da idade, a existência de comorbilidades é um fator importante na exequibilidade cirúrgica e eficácia a longo prazo. ${ }^{10,13} \mathrm{Na}$ presente série, o diagnóstico prévio de doença do SNC não teve impacto significativo na taxa de conversão para laparotomia ou no número de iatrogenias cirúrgicas, o que reforça a exequibilidade e segurança da cirurgia antirrefluxo por via laparoscópica nestas crianças.

A realização de procedimentos associados, nomeadamente colocação de botão de gastrostomia, foi significati- vamente superior na primeira infância e nas crianças com idade superior a três anos, o que está relacionado com a maior frequência de doença do SNC nestes grupos etários. A realização de gastrostomia não parece ser um fator independente na ocorrência de complicações, apesar de acarretar um aumento substancial da duração do procedimento laparoscópico, ${ }^{13,28}$ resultados confirmados nesta série. Nas crianças com doença do SNC verificou-se um aumento significativo nos dias de internamento que deverá estar correlacionado com a doença de base. Alguns autores descrevem um risco particularmente elevado de complicações pós-operatórias, piores resultados a longo prazo, maior risco de recorrência dos sintomas e maior mortalidade..$^{9,10,12,13,16,20}$ No entanto, estes resultados são díspares. ${ }^{10-12,16} \mathrm{Na}$ nossa série, com um seguimento pós-operatório de médio prazo, a cirurgia antirrefluxo em crianças com doença do SNC não teve maior frequência de recidiva clínica ou taxa de reoperação, apoiando o papel do tratamento cirúrgico da DRGE neste grupo de doentes.

Relativamente à $A E$ também é assumida uma menor eficácia do tratamento cirúrgico. ${ }^{11,13,20}$ Contudo, os dois casos de recidivas verificados na presente série ocorreram devido a herniação paraesofágica em crianças sem doença do SNC ou AE.

A realização de um procedimento de drenagem gástrica (piloroplastia /piloromiotomia), justificado pela alta prevalência de esvaziamento gástrico lento, particularmente em crianças com doença do SNC, é um aspeto controverso. ${ }^{6,21,27,29}$ Apesar do papel fisiopatológico do esvaziamento gástrico lento na DRGE, ${ }^{21,27,29}$ nenhum dos doentes fol submetido a piloroplastia/piloromiotomia, dado já ter sido demonstrado que o esvaziamento gástrico lento tende a normalizar após cirurgia antirrefluxo..$^{30,31}$

\section{CONCLUSÃO}

Os resultados da nossa série corroboram a exequibilidade, eficácia e segurança da cirurgia antirrefluxo por via laparoscópica no lactente e na primeira infância, assim como nas crianças com comorbilidades tais como doença do SNC ou antecedentes de AE.

\section{CONFLITO DE INTERESSES}

Os autores declaram que não houve conflito de interesses na realização deste trabalho.

\section{FONTES DE FINANCIAMENTO}

Os autores declaram a inexistência de fontes de financiamento externas.

\section{REFERÊNCIAS}

1. Campanozzi A, Boccia G, Pensabene L, Panetta F, Marseglia A, Strisciuglio $P$, et al. Prevalence and natural history of gastroesophageal reflux: pediatric prospective survey. Pediatrics. 2009;123:779-83.

2. Gold BD. Review article: epidemiology and management of gastro-oesophageal reflux in children. Aliment Pharmacol Ther. 2004;19:s22-7.

3. Sherman PM, Hassall E, Fagundes-Neto U, Gold BD, Kato S, Koletzko $\mathrm{S}$, et al. A global, evidence-based consensus on the definition of gastro- esophageal reflux disease in the pediatric population. Am J Gastroenterol. 2009;104:1278-95.

4. Vakil N. Disease definition, clinical manifestations, epidemiology and natural history of GERD. Best Pract Res Clin Gastroenterol. 2010;24:759-64.

5. Jeurnink SM, van Herwaarden-Lindeboom MY, Siersema PD, Fischer K Houwen RH, van der Zee DC. Barrett's esophagus in children: does it 
need more attention? Dig Liver Dis. 2011;43:682-7.

6. Estevão-Costa J. Tratamento cirúrgico do refluxo gastro-esofágico. Arq Port Cir. 2000;9:93-9.

7. Chawla S, Seth D, Mahajan P, Kamat D. Gastroesophageal reflux disorder: a review for primary care providers. Clin Pediatr. 2006;45:7-13.

8. Iwanaka T, Kanamori Y, Sugiyama M, Komura M, Tanaka Y, Kodaka T, et al. Laparoscopic fundoplication for gastroesophageal reflux disease in infants and children. Surg Today. 2010;40:393-7.

9. IPEG guidelines for the surgical treatment of pediatric gastroesophageal reflux disease (GERD). J Laparoendosc Adv Surg Tech A. 2009;19:sxxiii.

10. Lasser MS, Liao JG, Burd RS. National trends in the use of antireflux procedures for children. Pediatrics. 2006;118:1828-35.

11. Tovar JA, Luis AL, Encinas JL, Burgos L, Pederiva F, Martinez L, et al. Pediatric surgeons and gastroesophageal reflux. J Pediatr Surg. 2007;42:277-83.

12. Kane TD, Brown MF, Chen MK. Position paper on laparoscopic antireflux operations in infants and children for gastroesophageal reflux disease. J Pediatr Surg. 2009;44:1034-40.

13. Lobe TE. The current role of laparoscopic surgery for gastroesophageal reflux disease in infants and children. Surg Endosc. 2007;21:167-74.

14. Shah SR, Jegapragasan M, Fox MD, Prince JM, Segura BJ, Kane TD. A review of laparoscopic Nissen fundoplication in children weighing less than 5 kg. J Pediatr Surg. 2010;45:1165-8.

15. LaRiviere CA, Parimi C, Huaco JC, Acierno SA, Garrison MM, Goldin $A B$. Variations in preoperative decision making for antireflux procedures in pediatric gastroesophageal reflux disease: a survey of pediatric surgeons. J Pediatr Surg. 2011;46:1093-8.

16. Mauritz FA, van Herwaarden-Lindeboom MY, Stomp W, Zwaveling S, Fischer K, Houwen $\mathrm{RH}$, et al. The effects and efficacy of antireflux surgery in children with gastroesophageal reflux disease: a systematic review. J Gastrointest Surg. 2011;15:1872-8.

17. Esposito C, Montupet P, Reinberg O. Laparoscopic surgery for gastroesophageal reflux disease during the first year of life. J Pediatr Surg. 2001;36:715-7.

18. Kane TD. Laparoscopic Nissen fundoplication. Minerva Chir. 2009;64:147-57.

19. Tannuri AC, Tannuri U, Mathias AL, Velhote MC, Romao RL, Goncalves $M E$, et al. Gastroesophageal reflux disease in children: efficacy of Nis- sen fundoplication in treating digestive and respiratory symptoms. Experience of a single center. Dis Esophagus. 2008;21:746-50.

20. Pacilli M, Chowdhury MM, Pierro A. The surgical treatment of gastro-esophageal reflux in neonates and infants. Semin Pediatr Surg. 2005;14:34-41.

21. Estevão-Costa J, Dias JA, Campos M, Trindade E, Teixeira-Pinto A Carvalho JL. Can esophageal $\mathrm{pH}$ monitoring predict delayed gastric emptying? J Pediatr Surg. 2004;39:1537-40.

22. Pinheiro PF, Simoes e Silva AC, Pereira RM. Current knowledge on esophageal atresia. World J Gastroenterol. 2012;18:3662-72.

23. Sistonen SJ, Pakarinen MP, Rintala RJ. Long-term results of esophageal atresia: Helsinki experience and review of literature. Pediatr Surg Int. 2011;27:1141-9.

24. Hassall E. Esophagitis and Barrett esophagus: unifying the definitions and diagnostic approaches, with special reference to esophageal atresia. J Pediatr Gastroenterol Nutr. 2011;52:S23-6.

25. Sullivan PB. Gastrointestinal problems in the neurologically impaired child. Baillieres Clin Gastroenterol. 1997;11:529-46.

26. Pensabene L, Miele E, Del Giudice E, Strisciuglio C, Staiano A. Mechanisms of gastroesophageal reflux in children with sequelae of birth asphyxia. Brain Dev. 2008;30:563-71.

27. Estevão-Costa J. Gastric emptying and pediatric gastroesophagea reflux. In: Esposito C, Montupet P, Rothenberg S, editors. The Gastroesophageal Reflux in Infants and Children. Berlim: Springer-Verlag; 2004. p.135-45.

28. Kawahara H, Mitani Y, Nose K, Nakai H, Yoneda A, Kubota A, et al. Should fundoplication be added at the time of gastrostomy placement in patients who are neurologically impaired? J Pediatr Surg. 2010;45:23736.

29. Estevão-Costa J, Campos M, Dias JA, Trindade E, Medina AM, Carvalho JL. Delayed gastric emptying and gastroesophageal reflux: a pathophysiologic relationship. J Pediatr Gastroenterol Nutr. 2001;32:471-4.

30. Estevão-Costa J, Fragoso AC, Prata MJ, Campos M, Trindade E, Dias JA, et al. Gastric emptying and antireflux surgery. Pediatr Surg Int. 2011;27:367-71

31. Pacilli M, Pierro A, Lindley KJ, Curry JI, Eaton S. Gastric emptying is accelerated following laparoscopic Nissen fundoplication. Eur J Pediatr Surg. 2008;18:395-7. 


\section{Cirurgia Laparoscópica do Refluxo Gastroesofágico na Criança \\ Acta Med Port 2013:26:705-710}

Publicado pela Acta Médica Portuguesa, a Revista Científica da Ordem dos Médicos

Av. Almirante Gago Coutinho, 151

1749-084 Lisboa, Portugal.

Tel: +351218428 215

E-mail: submissao@actamedicaportuguesa.com

www.actamedicaportuguesa.com

ISSN:0870-399X | e-ISSN: 1646-0758

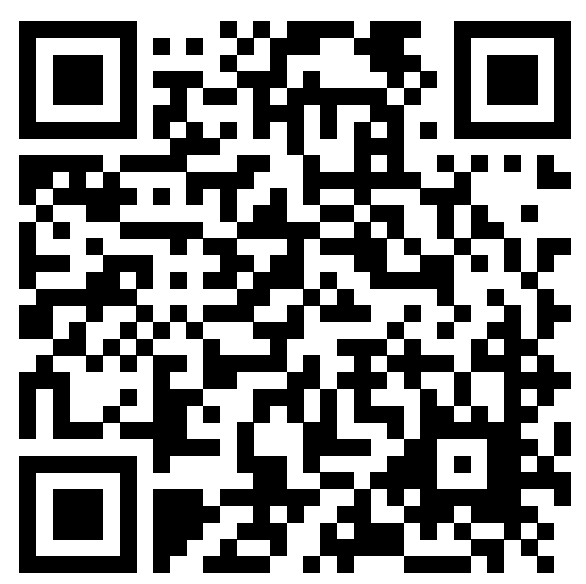

